\title{
Polarimetric analysis of the human cornea measured by polarization-sensitive optical coherence tomography
}

Félix Fanjul-Vélez

University of Cantabria

Electronics Technology, Systems and Automation Engineering Department

Applied Optical Techniques Group

Avenida de los Castros $\mathrm{S} / \mathrm{N}$

Santander, Cantabria 39005 Spain

Michael Pircher

Bernhard Baumann

Erich Götzinger

Christoph K. Hitzenberger

Medical University of Vienna

Center for Medical Physics and Biomedical Engineering

Währinger Straße 13

1090 Vienna, Austria

\section{José Luis Arce-Diego}

University of Cantabria

Electronics Technology, Systems and Automation Engineering Department

Applied Optical Techniques Group

Avenida de los Castros S/N

Santander, Cantabria 39005 Spain

\begin{abstract}
Corneal polarimetry measurement has been the object of several papers. The results of techniques like polarization-sensitive optical coherence tomography (PS-OCT), scanning laser polarimetry, or polarization microscopy are contradictory. Some studies propose a biaxial-like birefringence pattern, while others postulate that birefringence grows at corneal periphery. Several theoretical approaches were proposed for the interpretation of these measurements, but they usually lack accuracy and an adequate consideration of the nonnormal incidence on the tissue. We analyze corneal polarization effects measured by PS-OCT. In vivo and in vitro PS-OCT images of the human cornea are acquired. PS-OCT measurements are apparently not in agreement with the biaxial-like birefringence pattern. We present a polarimetric model of the human cornea based on the extended Jones matrix formalism applied to multilayered systems. We also apply the Poincaré equivalence theorem to extract optic axis orientation and birefringence. The results show that for a fibrils orientation pattern composed by alternating circular and radial fibrils, the birefringence is biaxial-like at the corneal center, and there is an almost circularly symmetric high-birefringence area at corneal periphery. The model could be useful for diagnosis of corneal diseases or corneal compensation in retinal polarimetric imaging. $\odot 2010$ Society of Photo-Optical Instrumentation Engineers. [DOI: 10.1117/1.3486540]
\end{abstract}

Keywords: corneal polarimetry; corneal birefringence; polarization-sensitive optical coherence tomography; extended Jones matrix; off-axis optical propagation.

Paper 10036RR received Jan. 25, 2010; revised manuscript received Jul. 14, 2010; accepted for publication Jul. 22, 2010; published online Sep. 7, 2010.

\section{Introduction}

The optical properties of the eye are especially suitable for the application of optical techniques. Polarimetric optical techniques such as polarization-sensitive optical coherence tomography ${ }^{1}$ (PS-OCT) or scanning laser polarimetry ${ }^{2}$ provide images with tissue-specific contrast. The different parts of the eye can be studied noninvasively by these techniques. One important tissue of the anterior chamber of the eye is the cornea. Corneal diagnosis requires an adequate knowledge of its polarimetric response to distinguish pathological or healthy states. It also influences the measurements of deeper structures in the eye fundus such as the retina. As the cornea is the first tissue to be traversed by optical radiation, polarimetric images must be compensated. ${ }^{3,4}$

Many papers were devoted to the study of corneal structure. Histology shows that the cornea is a layered tissue. ${ }^{5}$ The nonkeratinized squamous epithelium is the first layer, starting from the outside. The next layer is Bowman's membrane, which makes a border with corneal stroma. This corneal stroma is formed mainly by layers of collagen fibrils. The

Address all correspondence to: Félix Fanjul-Vélez, University of Cantabria, Electronics Technology, Systems and Automation Engineering Department, Applied Optical Techniques Group, Avenida de los Castros S/N, Santander (Cantabria), Spain 39005. Tel: 34-942-20-15-45; Fax: 34-942-20-18-73. E-mails: fanjulf@unican.es following layers are Descemet's membrane and the corneal endothelium. The latter is a unicellular layer composed predominantly of mitochondria. The dominant layer in the cornea is the corneal stroma, which makes up most of the corneal thickness. As a consequence, the polarization properties of the cornea depend mainly on the stroma. Collagen fibril organization was studied by means of electron microscopy ${ }^{6}$ and $\mathrm{x}$-ray diffraction. ${ }^{7,8}$ The results show that fibrils are disposed in layers, where they maintain a particular orientation. In successive layers, the orientation changes in an arbitrary way, but in general fibrils in successive layers are thought to be oriented at nearly orthogonal angles, although there are also some fibrils with a 45-deg angle. Some interlaced fibrils go from one layer to the next. These histological or electron microscopy images provide information on the microscopic structure. However, the corneal polarization behavior analysis would require a macroscopic approach.

The cornea is composed of fibrils, whose orientation is not totally clear, so it presents a birefringent form behavior. As a consequence, polarization optical techniques are particularly useful for the study of the cornea. Several polarization techniques were used to image the cornea: Jones polarimetry, ${ }^{9}$ Mueller polarimetry, ${ }^{10}$ polarization microscopy,${ }^{11}$ or scanning

1083-3668/2010/15(5)/056004/10/\$25.00 @ 2010 SPIE 
laser polarimetry. ${ }^{12}$ From the results obtained, the authors assume a biaxial or biaxial-like optical behavior. This was first proposed by Van Blokland and Verhelst, ${ }^{13}$ who used Mueller matrix ellipsometry. The birefringent properties of the cornea were also measured ${ }^{14}$ by PS-OCT. The results obtained did not apparently coincide with the biaxial-like behavior. PSOCT shows low retardation in the corneal centre and a high birefringence at the corneal periphery. This is also reported in other papers. ${ }^{15,16}$ As a consequence, there is a contradiction in the experimental results.

Previous approaches tried to explain the experimental optical behavior of the cornea with a particular model. Only some of them proposed a qualitative fibrils orientation pattern. In this paper, we employ an a priori model of corneal structure that tries to predict the corneal polarization response. A layered model based on on-axis Jones retarders with varying optic axis orientation was previously proposed. ${ }^{17}$ We also presented a study with a similar model and different fibrils arrangements. ${ }^{18}$ In both approaches, light must be perpendicular to the corneal surface and fibrils must be contained in a plane perpendicular to the incident radiation. The model proposed here is based on the extended Jones matrix formalism. This enables the consideration of nonnormal incidence and arbitrary fibril orientation. ${ }^{19}$ The Poincaré equivalence theorem is applied to extract optic axis orientation and retardation at each point. The polarimetric approach is presented in Sec. 2. Section 3 explains the PS-OCT systems employed for the measurement of in vitro and in vivo images of the human cornea. In Sec. 4, the model results are compared with the measurements, and the implications are discussed. Section 5 presents the conclusions.

\section{Polarization Model}

The models used in the already mentioned papers to interpret the results of the different optical techniques applied to the cornea present limitations. The main one is related with the assumption that the incident radiation must be perpendicular to the tissue surface and also that the collagen fibrils of the birefringent tissue must lie in a plane parallel to this surface. Assuming these limitations, the tissue is usually modeled by a Jones-equivalent linear retarder with normal incidence and optic axis in the direction of the collagen fibrils. A further decomposition or numerical adjustment procedure extracts optic axis orientation and retardation, either cumulative ${ }^{20}$ or depth resolved. ${ }^{21}$ In the case of the cornea, light is usually incident with a nonperpendicular angle due to corneal curvature. As stated in the introduction, the fibrils of the cornea are not always contained in a plane perpendicular to the incident light direction. In a previous paper, ${ }^{18}$ we took into account corneal curvature by the cosine of the angle between the fibrils and the plane perpendicular to the incident direction. This approach was too simplistic to show the complex birefringent corneal response. The results did not match the experimental measurements completely.

Nonnormal-incidence and/or nonparallel-to-the-surface fibrils require the use of approaches that take into account birefringent propagation of light explicitly. Off-axis propagation of light in birefringent crystals was studied by $\mathrm{Yeh}^{22}$ and $\mathrm{Gu}$ and Yeh. ${ }^{23}$ They proposed a $2 \times 2$ matrix method that extends the Jones calculus to this general case of nonnormal

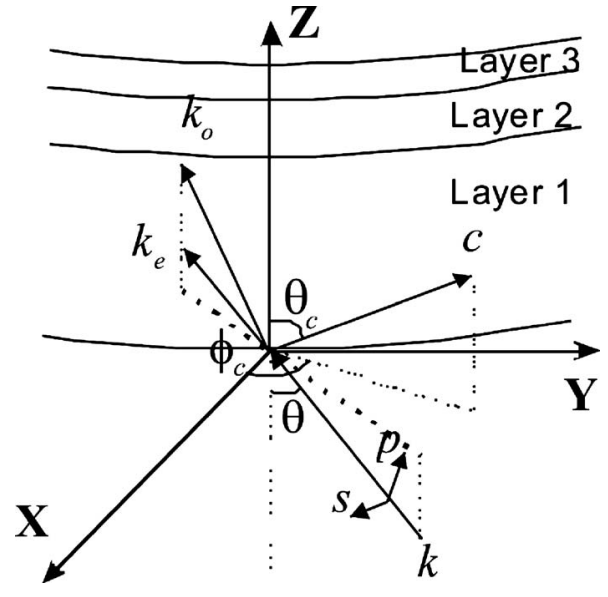

Fig. 1 Main crystal parameters. The principal reference system at each point is $X Y Z$. The layers of the birefringent tissue are parallel to the $X Y$ plane at the incidence point. In the case of the cornea, these layers are curved and $\mathbf{k}$ is the incident propagation vector, $\mathbf{k}_{o}$ and $\mathbf{k}_{e}$ are the ordinary and extraordinary propagation vectors inside the birefringent structure; $\mathbf{c}$ is the optic axis (polar angle $\theta_{C}$, azimuthal angle $\left.\phi_{C}\right)$; and $\mathbf{s}$ and $\mathbf{p}$ define the local polarization reference system.

incidence. This method was used for the calculus of the propagation of light through two and three layers of anisotropic materials in which the optic axis is constrained to be parallel to the layers, and in which the effects at the boundaries are not taken into account. ${ }^{24}$ Some attempts to apply it to biological tissues imposed restrictions in the orientation of the optic axis or the incident light, or considered only one propagation vector. ${ }^{25}$ In this paper, we will use the extended Jones matrix method with no such restrictions ${ }^{19}$ in the particular case of the cornea.

The cornea is a birefringent collagen-based biological tissue. This kind of tissue can be modeled as a positive uniaxial crystal. The optic axis of this crystal is coincident with the fibrils direction. The main parameters of the crystal can be seen in Fig. 1. The boundary of the crystal is on the $X Y$ plane of the $X Y Z$ principal coordinate system. A uniaxial crystal is defined by the extraordinary $n_{e}$ and ordinary $n_{o}$ refractive indices corresponding to the fibril direction and to the perpendicular to it, respectively, and by the orientation of the optic axis $\mathbf{c}$, described with the polar angle $\theta_{C}$ and azimuthal angle $\phi_{C}$. The electromagnetic propagation of the incident light is described by the propagation vector $\mathbf{k}$. The two propagation vectors $\mathbf{k}_{o}$ (ordinary beam) and $\mathbf{k}_{e}$ (extraordinary beam) inside the uniaxial medium can be calculated by ${ }^{22,23}$

$$
\begin{gathered}
k_{o z}=\left[\left(\frac{n_{o} \omega}{c}\right)^{2}-k_{x}^{2}-k_{y}^{2}\right]^{1 / 2}, \\
k_{e z}=\frac{B+\left(B^{2}-4 A C\right)^{1 / 2}}{2 A},
\end{gathered}
$$

where $k_{o z}$ and $k_{e z}$ are the $Z$ components of the propagation vectors. In these equations, $\omega$ is the angular frequency; $c$ is the speed of light in vacuum; and parameters $A, B$, and $C$ are given by 


$$
\begin{gathered}
A=\frac{\sin ^{2} \theta_{C}}{n_{e}^{2}}+\frac{\cos ^{2} \theta_{C}}{n_{o}^{2}}, \\
B=\left(k_{x} \cos \phi_{C}+k_{y} \sin \phi_{C}\right) \sin \left(2 \theta_{C}\right)\left(\frac{1}{n_{e}^{2}}-\frac{1}{n_{o}^{2}}\right), \\
C=\frac{\left(k_{x} \cos \phi_{C}+k_{y} \sin \phi_{C}\right)^{2} \cos ^{2} \theta_{C}+\left(-k_{x} \sin \phi_{C}+k_{y} \cos \phi_{C}\right)^{2}}{n_{e}^{2}}+\frac{\left(k_{x} \cos \phi_{C}+k_{y} \sin \phi_{C}\right)^{2} \sin ^{2} \theta_{C}}{n_{o}^{2}}-\left(\frac{\omega}{c}\right)^{2} .
\end{gathered}
$$

The corneal stroma is a layered tissue and each layer has a different preferential fibril orientation. The orientation of the corneal fibrils changes with position in the cornea, as explained in the introduction. A stack of layers with different optic axis orientations is defined at each position by extended Jones matrices. The layers in the stack must be parallel to each other, as represented in Fig. 1. This is the case of corneal stroma. Small birefringence, $\left|n_{e}-n_{o}\right|<<n_{o}, n_{e}$ will be assumed. Dichroism, optical activity, and multiple reflections are neglected. Under these conditions, the extended Jones matrix formalism can be written as:

$$
\begin{aligned}
\mathbf{E}_{\text {out }} & =\mathbf{L}_{T} E_{\text {in }} \\
& =\mathbf{B}_{\text {fin }} \mathbf{P}_{N} \mathbf{T}_{N-1, N} \mathbf{P}_{N-1} \mathbf{T}_{N-2, N-1} \ldots \mathbf{T}_{2,3} \mathbf{P}_{2} \mathbf{T}_{1,2} \mathbf{P}_{1} \mathbf{B}_{\mathrm{ini}} \mathbf{E}_{\mathrm{in}}
\end{aligned}
$$

The first matrix (from right to left) $\mathbf{B}_{\text {ini }}$ and the last $\mathbf{B}_{\text {fin }}$ model the interaction between an isotropic external medium (usually air) and the biological tissue boundary. The $\mathbf{P}_{i}$ matrices represent the propagation of radiation through the $i$ 'th layer, according to the specific fibril orientation. The $\mathbf{T}_{i, i+1}$ matrices take into account the propagation between uniaxial layers $i$ and $i+1$. The incident vector is represented by the Jones vector $\mathbf{E}_{\text {in }}$, while the output Jones vector after passing the complex medium is $\mathbf{E}_{\text {out }}$. The matrix product $\mathbf{L}_{T}$ represents the extended Jones matrix of the biological tissue. In this analysis, attention must be paid to the reference system. We consider that the incident and output Jones vectors are referred to a system defined by the polarization states of TE (transverse electric) and TM (transverse magnetic) incident vectors $\mathbf{s}$ and $\mathbf{p}$, respectively (Fig. 1). We also define polarization vectors $\mathbf{o}$ and $\mathbf{e}$ as the polarization states of the ordinary and extraordinary waves. Here $\mathbf{p}_{o}$ is a vector perpendicular to both vectors $\mathbf{s}$ and $\mathbf{k}_{o}\left(\mathbf{p}_{o}=\mathbf{k}_{o} \times \mathbf{s}\right)$. The matrices can be expressed as

$$
\begin{gathered}
\mathbf{B}_{\mathrm{ini}}=\left(\begin{array}{cc}
\mathbf{s} \cdot \mathbf{o} t_{s} & \mathbf{p}_{o} \cdot \mathbf{o} t_{p} \\
\mathbf{s} \cdot \mathbf{e} t_{s} & \mathbf{p}_{o} \cdot \mathbf{e} t_{p}
\end{array}\right) \\
\mathbf{B}_{\mathrm{fin}}=\left(\begin{array}{cc}
\mathbf{o} \cdot \mathbf{s} t_{s}^{\prime} & \mathbf{s} \cdot \mathbf{e} t_{s}^{\prime} \\
\mathbf{p}_{o} \cdot \mathbf{o} t_{p}^{\prime} & \mathbf{p}_{o} \cdot \mathbf{e} t_{p}^{\prime}
\end{array}\right) \\
\mathbf{P}_{i}=\left(\begin{array}{cc}
\exp \left(-j k_{o z} d_{i}\right) & 0 \\
0 & \exp \left(-j k_{e z} d_{i}\right)
\end{array}\right)
\end{gathered}
$$

$$
\mathbf{T}_{i, i+1}=\left(\begin{array}{ll}
\mathbf{o}_{i} \cdot \mathbf{o}_{i+1} & \mathbf{e}_{i} \cdot \mathbf{o}_{i+1} \\
\mathbf{o}_{i} \cdot \mathbf{e}_{i+1} & \mathbf{e}_{i} \cdot \mathbf{e}_{i+1}
\end{array}\right) .
$$

In these matrices $d_{i}$ is the thickness of the $i$ 'th layer, and $t_{s}, t_{p}$, $t_{s}^{\prime}$, and $t_{p}^{\prime}$ are the Fresnel transmission coefficients. The application of this system to the cornea requires the definition of auxiliary coordinate systems. They are represented in Fig. 2. A local reference system is defined by vectors $\mathbf{r}, \boldsymbol{\theta}$, and $\boldsymbol{\phi}$, corresponding to the spherical coordinate vectors at each point of the corneal surface. The angle of incidence is calculated with respect to vector $\mathbf{r}$. The results of the extended Jones matrix method are referred to the system defined by vectors $\mathbf{S}$ and $\mathbf{p}$. They depend on the position. The final representation coordinate system is defined by the $X^{\prime} Y^{\prime} Z^{\prime}$ system. Note that $Z^{\prime}$ has the same direction as the incident vector, and $Y^{\prime}$ is chosen to be perpendicular to vector $\mathbf{X}$ of the principal reference system.

PS-OCT usually measures the cumulative slow axis orientation and retardation of the sample, which would correspond to an equivalent retarder. The algorithm of the experimental setup normally assumes that the biological tissue can be modeled as a linear Jones retarder. ${ }^{20}$ However, the measured signals do not correspond to that assumption in general. The extended Jones matrix better resembles the measured signals due to the nonnormal incidence and arbitrary fibril orientation. The axis orientation and retardation are calculated assuming that the matrix is that of a retarder, in the same way as

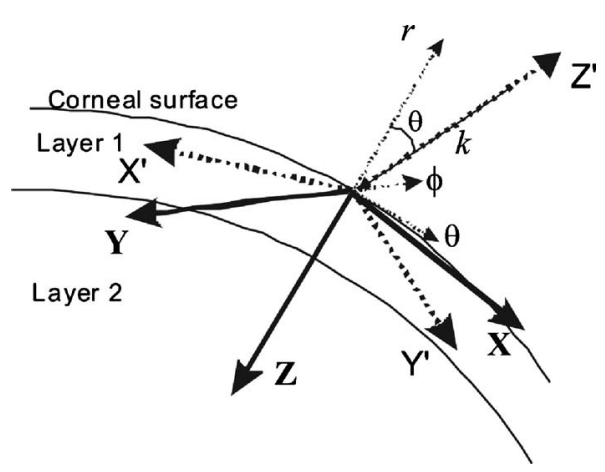

Fig. 2 Coordinate systems used in the analysis, where $X Y Z$ is the principal coordinate system. The local coordinate system is defined by vectors $\mathbf{r}, \boldsymbol{\theta}$, and $\boldsymbol{\phi}$, corresponding to the spherical coordinate vectors; $\theta$ is the angle of incidence; $X^{\prime} Y^{\prime} Z^{\prime}$ is the final representation coordinate system; vectors $\mathbf{Z}^{\prime}$ and $\mathbf{k}$ have the same direction; and $\mathbf{Y}^{\prime}$ is perpendicular to vector $\mathbf{X}$. 
in the experimental algorithm. ${ }^{20}$ The Poincaré equivalence theorem $^{26}$ is applied to the extended Jones matrix $\mathbf{L}_{T}$ by means of the group theory: ${ }^{27}$

$$
\mathbf{L}_{T}=\mathbf{R}\left(-\theta_{T}\right) \mathbf{D}(\delta) \mathbf{R}\left(\theta_{T}\right) \mathbf{R}\left(\theta_{R}\right) .
$$

In this equation, matrices $\mathbf{R}(\theta)$ and $\mathbf{D}(\delta)$ are a general rotator of angle $\theta$ and a retarder of retardation $\delta$, respectively. These are the equivalent retarder and rotator of the extended Jones matrix of the cornea at the position of interest. Also, $\theta_{T}$ is the equivalent slow optic axis orientation, $\delta$ is the equivalent retardation of the sample, and $\theta_{R}$ is the equivalent rotation. This equation represents the parametrisation of a unimodular $\mathrm{SU}(2)$ matrix. It enables the calculation of an equivalent retarder and a rotator. The parameters of the retarder $\theta_{T}$ and $\delta$ are equivalent to PS-OCT measured values. The algorithm presents ambiguities due to the periodicity of the angles involved. The implementation used here limits the variation range to try to avoid these ambiguities. The optic axis orientation is constrained to be in the interval $[0, \pi)$. Orientations in $[\pi, 2 \pi)$ are equivalent. The retardation is in the range $[0, \pi / 2]$, and as a consequence greater values will be wrapped to this interval.

\section{PS-OCT Measurements}

PS-OCT is a functional extension of OCT that enables the extraction of polarization properties of tissues. ${ }^{1,28}$ Several variants of PS-OCT have been reported; the instruments used in this paper are based on illumination of the sample with circularly polarized light and a phase sensitive light detection in two orthogonal polarization channels. ${ }^{20}$ A single measurement per sample location provides reflectivity, retardation, and optic axis orientation.

The in vitro data are taken from a previous study, ${ }^{14}$ where the measurements were performed with a slow time domain version of PS-OCT. Details on the instrument and the sample preparation can be found in Ref. 14. The instrument used a superluminescent diode (SLD) with a center wavelength of $828 \mathrm{~nm}$ and a bandwidth of $22.4 \mathrm{~nm}$, providing a theoretic depth resolution of $13.5 \mu \mathrm{m}$ in air or $\sim 10 \mu \mathrm{m}$ in tissue (assuming a group index of $n_{g}=1.35$ ). The 3-D data sets of excised corneas were obtained by recording of $A$-scans $(z)$ on a 2-D $(x-y)$ lateral matrix on the sample. A 3-D data set consisted of $80 \times 80 A$-scans (covering $8 \times 8 \mathrm{~mm}^{2}$ ) of $2.5-\mathrm{mm}$ length. Reflectivity $R$, retardation $\delta$, and slow axis orientation $\theta$ were calculated from the amplitudes and phase differences of the horizontal and vertical polarization channels, as described in detail in Ref. 14. The unambiguous ranges of $\delta$ and $\theta$ were 0 to 90 and 0 to $180 \mathrm{deg}$, respectively [note that the algorithm used to calculate $\theta$ causes a 90 -deg change in $\theta$ at depth positions $z$ where $\delta$ crosses $90 \mathrm{deg}$ (or multiples of 90 deg)].

In vivo data were recorded in healthy volunteers after informed consent was obtained. For these measurements, a spectral domain (SD) PS-OCT instrument was used (cf. Fig. 3 ). The instrument was initially developed for retinal imaging, and details of its principle and operation can be found in Ref. 29. Therefore, only a short description is provided here, including the main changes implemented for anterior segment imaging. An SLD with center wavelength of $840 \mathrm{~nm}$ and

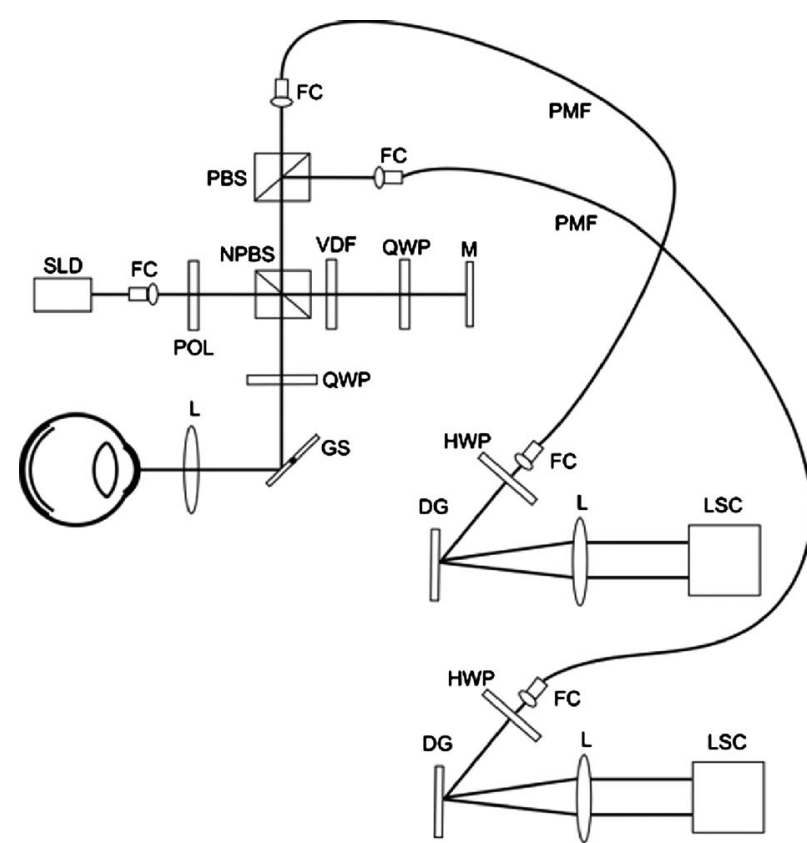

Fig. 3 Sketch of SD PS-OCT system used for in vivo imaging: SLD, superluminescent diode; FC, fiber coupler; POL, polarizer; NPBS, nonpolarizing beamsplitter; VDF, variable neutral density filter; QWP, quarter-wave plate; $M$, mirror; $G S$, galvo scanner; L, lens; PBS, polarizing beamsplitter; PMF, polarization-maintaining fiber; HWP, half wave plate; DG, diffraction grating; and LSC, line scan camera.

bandwidth of $50 \mathrm{~nm}$ was used, providing a theoretic depth resolution of $6.2 \mu \mathrm{m}$ in air and $\sim 4.6 \mu \mathrm{m}$ in tissue. The beam illuminates, after being vertically polarized, a Michelson interferometer, where the beam is split by a nonpolarizing beamsplitter (NPBS) into a reference and a sample beam. The reference beam passes a variable neutral density filter (VDF) and a quarter-wave plate (QWP), oriented at $22.5 \mathrm{deg}$ to the horizontal. After reflection at the reference mirror and backpropagating through the QWP, the reference beam is in a linear polarization state oriented at $45 \mathrm{deg}$ to provide equal reference power in both the horizontal and the vertical polarization states.

The sample beam transits a QWP oriented at $45 \mathrm{deg}$, providing circularly polarized light to the sample. An $x-y$ galvanometer scanner (GS) scans the beam over the sample. For corneal and anterior segment imaging, the following changes were made to the scanning unit, as compared to the previous retinal scanner: (1) the two-lens telescope of the retinal scanning unit was replaced by a single achromatic lens $\mathrm{L}$ that is positioned at its focal distance from the fast $(x)$ scanner's pivot point; and (2) the $x$ scanner is illuminated in an off-pivot point configuration. This introduces ${ }^{30}$ a phase shift during the $x$ scan, enabling full-range complex SD-OCT that doubles the axial measurement range to $\sim 6 \mathrm{~mm}$ (in air), enabling us to image the full anterior chamber depth without mirror artefacts. In the detection arm, a polarizing beamsplitter (PBS) separates the interfering beams into horizontal and vertical polarization components that are guided by polarizationmaintaining fibers to two identical spectrometers that record the orthogonal polarization channels in parallel. 


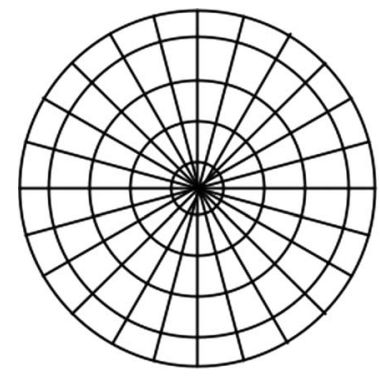

(a)

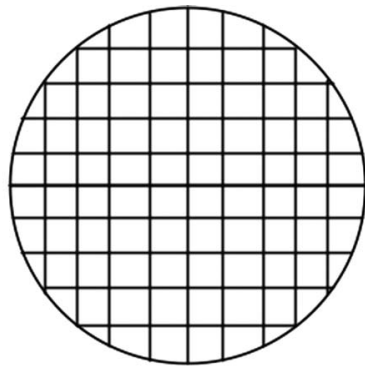

(b)

Fig. 4 Fibril orientation diagram of the different configurations (en face view): (A) circular-radial pattern; and (B) horizontal-vertical pattern. Corneal apex is in the center of the image. Fibrils that belong to different layers are represented together at the plane of the paper.

The instrument was operated at $20 \mathrm{kA}$ scans $/ \mathrm{s}$ and achieved a sensitivity of $98 \mathrm{~dB}$. For a 3-D data set, 60 $B$-scans in horizontal $(x)$ direction (each consisting of 1000 $A$-scans) were recorded separated equidistantly in $y$ direction. To account for the unequal sampling densities in $x$ and $y$ directions, data were interpolated in the $y$ direction to obtain square-shaped images. Unambiguous ranges of $\delta$ and $\theta$ are similar to that of the time domain instrument (the same comment on 90-deg jumps of $\theta$ applies).

Because of the better signal quality, the in vivo polarization patterns displayed in the results section were obtained at the iris and lens surface rather than at the posterior corneal surface. The dispersion induced change of the coherence length within the aqueous is estimated ${ }^{31}$ to be only $\sim 8 \%$, based on the light source parameters used in the measurements and assuming an average anterior chamber depth of $3.6 \mathrm{~mm}$ according to Gullstrand's schematic eye. Furthermore, both polarization channels are affected equally by dispersion since the aqueous is not birefringent. Therefore, the patterns in the iris/lens plane are similar to those at the posterior cornea ${ }^{32}$ [we confirmed the similarity by comparison to the (noisier) posterior corneal surface patterns; results are not shown here].

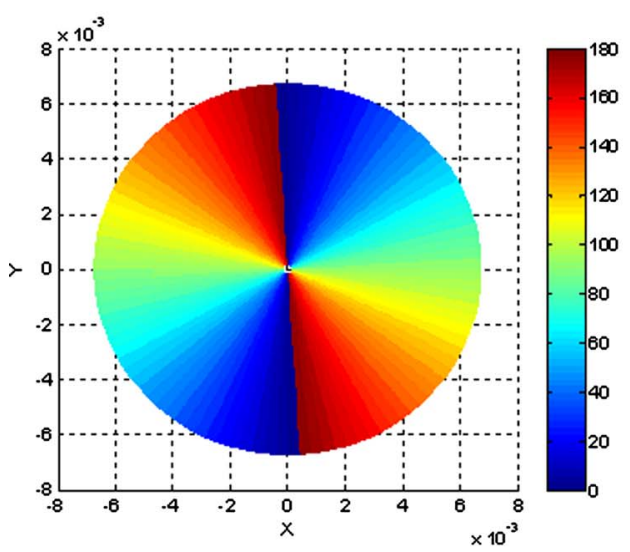

(a)

\section{Results and Discussion}

The polarimetric model was applied to the cornea. The experimental setups described in the previous section give results for one-way polarization parameters. ${ }^{20}$ As a consequence, taking into account the reversibility conditions, the one-way extended Jones matrix was calculated following the theory in Sec. 2. Retardation and axis orientation distribution at the posterior corneal surface were calculated. The stroma was assumed to be composed of 200 layers, with a total thickness ${ }^{5}$ of $0.52 \mathrm{~mm}$. The corneal radius was assumed to be $7.8 \mathrm{~mm}$. The corneal birefringence value, that is, the difference between the extraordinary and ordinary refractive indices, is ${ }^{13}$ around $10^{-3}$. The incident radiation was assumed to be perpendicular to the corneal surface at the apex. The fibril orientation pattern in the cornea is controversial, as stated in the introduction. Several configurations were postulated in previous work. We chose two patterns for simulation that are in agreement with the microscopic observation of local (approximately) perpendicular fibril orientation in successive layers and that are also reasonable from a biomechanical point of view. The configurations chosen are preferentially oriented radial and circular fibrils and preferentially oriented horizontal and vertical fibrils. Other configurations ${ }^{18}$ (preferentially oriented circular and vertical fibrils, and preferentially oriented fibrils in a cross-arc pattern) were also tested. The results (not shown here) are not in agreement with the experiments, especially the slow optic axis orientation. The two selected patterns are schematically represented in Fig. 4. The results for the slow optic axis orientation and retardation (at the posterior corneal surface) are shown in Figs. 5 and 6. The slow-axis orientation pattern is dependent on the fibril orientation, as expected [Figs. 5(a) and 6(a)]. In both cases the slow axis orientation varies spatially following a circle centred at the corneal apex. The variation is smooth, and it shows all the possible angular values. In Fig. 5(a) (circular-radial pattern) the angles are constant along the radial direction, and the 0 -deg angle is located at the vertical direction. In the horizontal-vertical case [Fig. $6(\mathrm{a})]$, the angles are notconstant along the radial direction, and there is a 90-deg rotation with respect to the previous

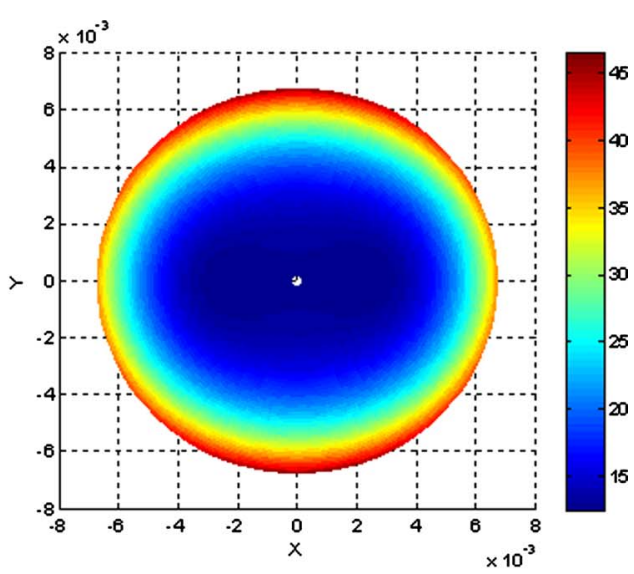

(b)

Fig. 5 Calculated polarization parameters at posterior corneal surface: (a) slow axis orientation and (b) retardation for the circular-radial case. Color scale: degrees. (Color online only.) 


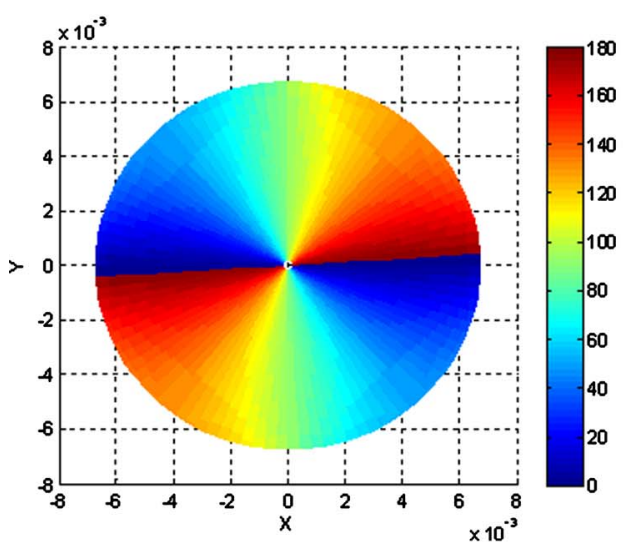

(a)

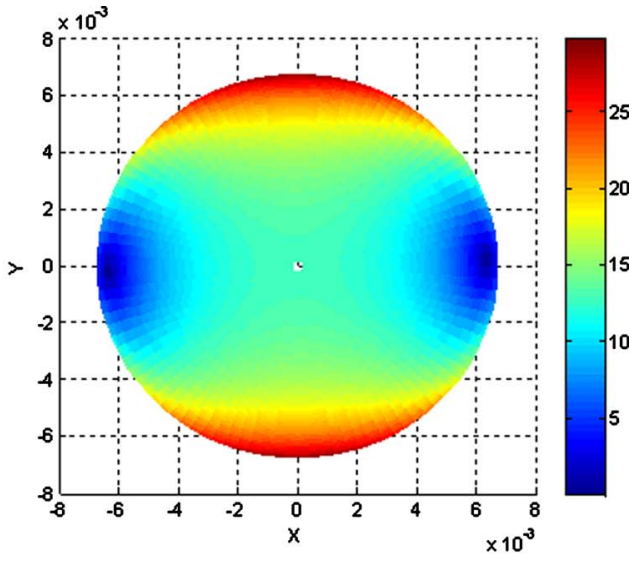

(b)

Fig. 6 Calculated polarization parameters at posterior corneal surface: (a) slow axis orientation and (b) retardation for the horizontal-vertical case. Color scale: degrees. (Color online only.)

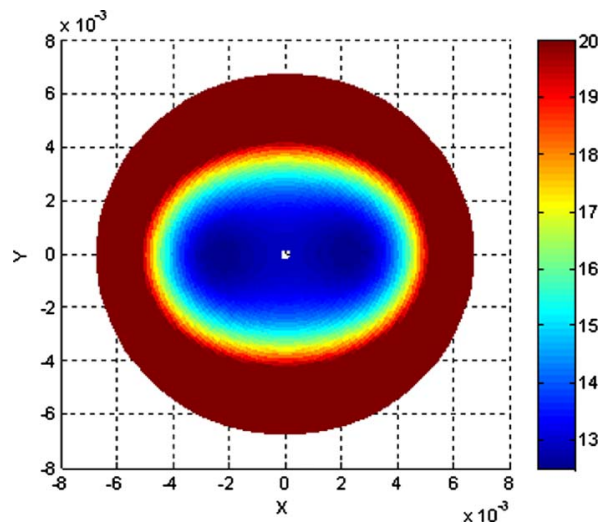

Fig. 7 Rescaled retardation pattern of the circular-radial case. The biaxial-like behavior can be appreciated. Values over 20 deg are constantly dark red. Color scale: degrees. (Color online only.)

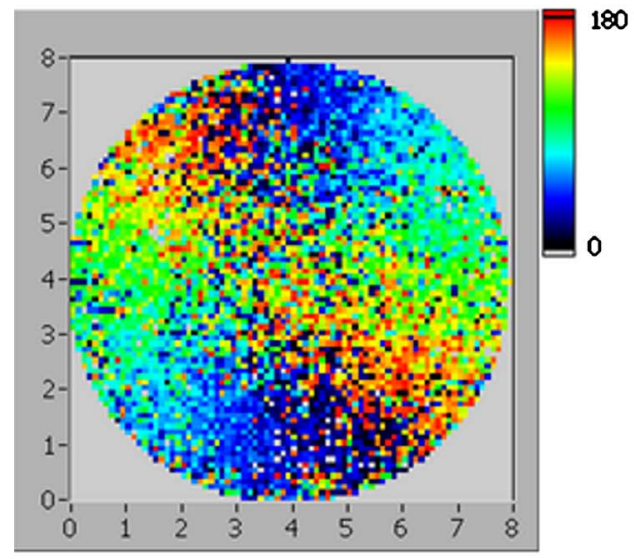

(a) distribution (0-deg angle follows the horizontal direction). Both retardation patterns [Figs. 5(b) and 6(b)] present in general a biaxial-like behavior, in agreement with several studies. ${ }^{9-13}$ There are two areas of low retardation that would correspond to the optic axes of the crystal clearly visible in Fig. 6(b). This is the expected behavior of a stack of birefringent uniaxial layers with varying incidence angle. ${ }^{24}$ In the circular-radial case [Fig. 5(b)] this biaxial-like behavior is not so evident due to the retardation increase that is produced at the corneal periphery. These increased values hide the biaxiallike pattern of the corneal central area, because a different color scale is used [in Fig. 5(b) the maximum of the color bar is around 50 deg, while in Fig. 6(b) it is only around $30 \mathrm{deg}$ ]. If this scale is readapted, the biaxial-like central pattern is visible (Fig. 7).

The distribution of polarization parameters at the posterior surface of the cornea was measured by PS-OCT, as described in Sec. 3. Figure 8 shows in vitro results that were taken from a previous study ${ }_{1}^{14}$ where the older time domain PS-OCT system was used. Figure 9 shows results of in vivo measurements obtained by the spectral domain system ${ }^{29}$ in the iris lens plane

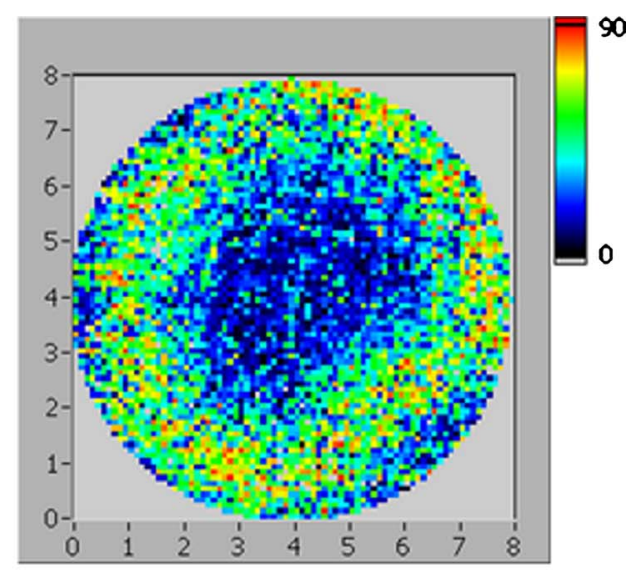

(b)

Fig. 8 (a) Slow-axis orientation and (b) retardation of the in vitro back surface of the cornea measured by PS-OCT; $x$ and $y$ axes in millimeters; color scale in degrees. (Images taken from Ref. 14.) (Color online only.) 


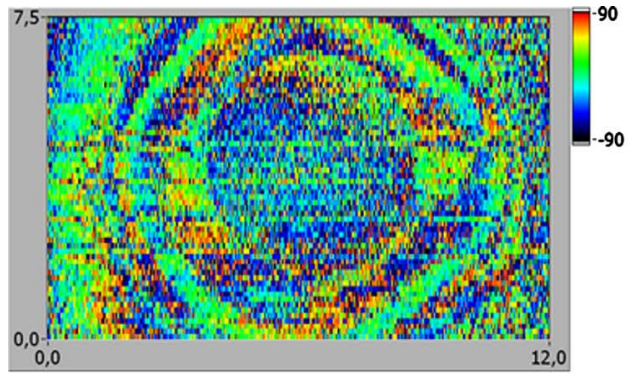

(a)

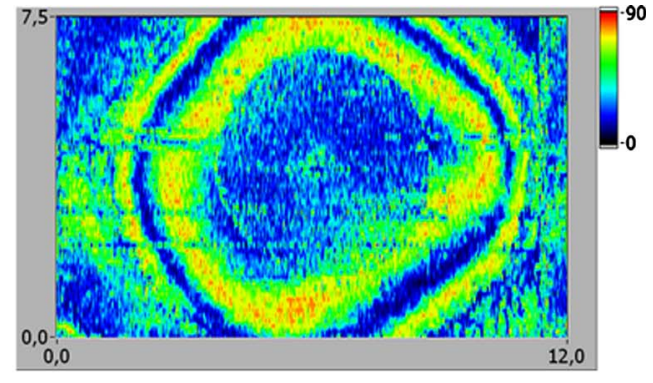

(b)

Fig. 9 (a) Slow-axis orientation and (b) retardation of the in vivo cornea measured at iris level by PS-OCT; $x$ and $y$ axes in millimeters; color scale in degrees. (Color online only.)

(see Sec. 3). The general behavior of the optic axis orientation shows a continuous rotation. This rotation goes from 0 to $180 \mathrm{deg}$ clockwise. This cycle appears twice along the whole circle. The in vivo slow-axis image is particularly noisy, but the general behavior seems to follow the previous analysis (if the color jumps at regions where the retardation crosses $90 \mathrm{deg}$ or multiples thereof are accounted for). The retardation patterns show a low retardation at the corneal center. The retardation is increased at the corneal periphery. In the in vivo images, the retardation increase at the periphery shows oscillations and a not completely circular pattern. The oscillations are due to the 90-deg wrapping of the algorithm. The noncircular pattern is supposed to be caused by the stress provoked by the eye muscles that allow ocular globe movement. This stress would provoke a change in the fibril pattern. The photoelastic effect would lead to other ellipsoid of indices. In the in vitro image, this stress is no longer present, so the pattern shape is almost circular.

The simulation results were obtained directly from the assumption of a particular fibril orientation arrangement. The slow-axis orientation of the simulated configurations [Figs. 5(a) and 6(a)] shows that the horizontal-vertical pattern starts from 0 deg at the $x$ axis, while the circular-radial case starts at $90 \mathrm{deg}$. As a consequence, there is a 90-deg rotation of the pattern. According to the experimental results [Fig. 8(a)], the circular-radial configuration resembles better the measured pattern. Retardation patterns in the horizontal-vertical case

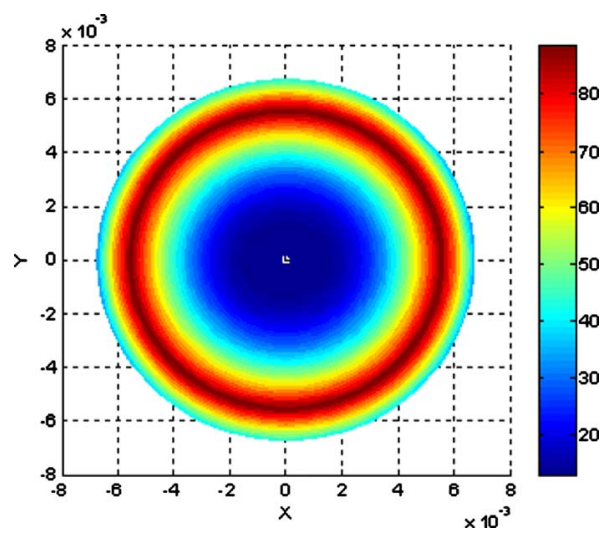

Fig. 10 Calculated corneal retardation pattern with increased birefringence (circular-radial fibril orientation). Color scale: degrees. (Color online only.)
[Fig. 6(b)] and in the circular-radial case [Fig. 5(b)] are biaxial-like. In the circular-radial case this behavior is not as obvious, and a rather circular increased retardation at the periphery is shown, as stated before. The biaxial-like behavior is present in the corneal center, as previously shown in Fig. 7. The experimental measurements of Figs. 8(b) and 9(b) are more in agreement with the circular-radial case. The main criticism of the PS-OCT corneal measurements was that the retardation pattern does not show a biaxial-like behavior, as reported in several papers. This was postulated to be caused by the lack of sensibility of the PS-OCT compared with other techniques. ${ }^{12}$ In these papers, the authors used a biaxial model to match a posteriori the experimental measurements. Fibril arrangements are also proposed from the experimental results. In this study, a layered geometry with varying fibril patterns is proposed a priori and the polarization response is predicted. The biaxial-like behavior comes directly from the structure proposed, it is not assumed. The polarimetric prediction can match the measurements with the tissue structure. This structure is usually related with the pathology of the tissue. As a consequence, although the biaxial models remain valid, this $a$ priori approach may be more useful for structure-related diagnosis. The circular-radial pattern presents a biaxial-like behavior, but only in the central part of the cornea (Fig. 7). This is the corneal area that is frequently measured. As a consequence, the circular-radial configuration is compatible with a biaxial-like behavior at the corneal center (in agreement with the mentioned previous papers), and it also resembles the

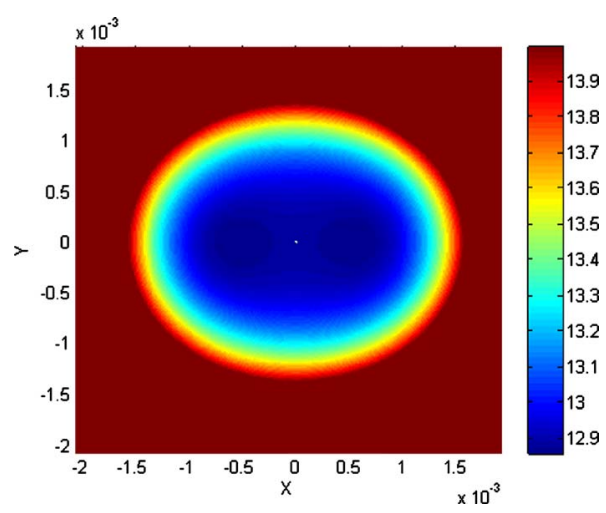

Fig. 11 Rescaled and zoomed version of Fig. 10. Values over $14 \mathrm{deg}$ are constantly dark red. Color scale: degrees. (Color online only.) 


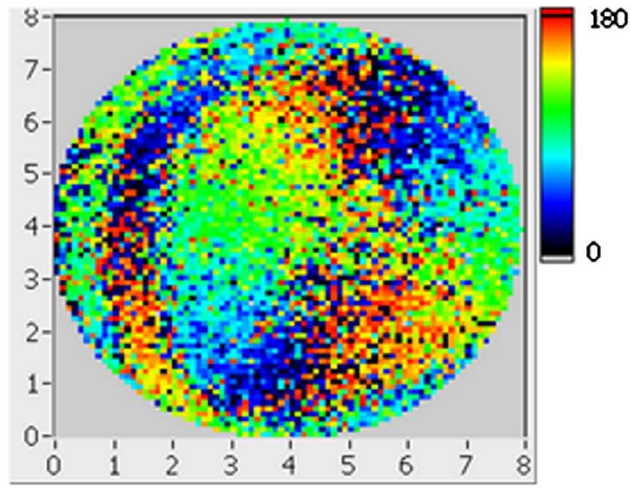

(a)

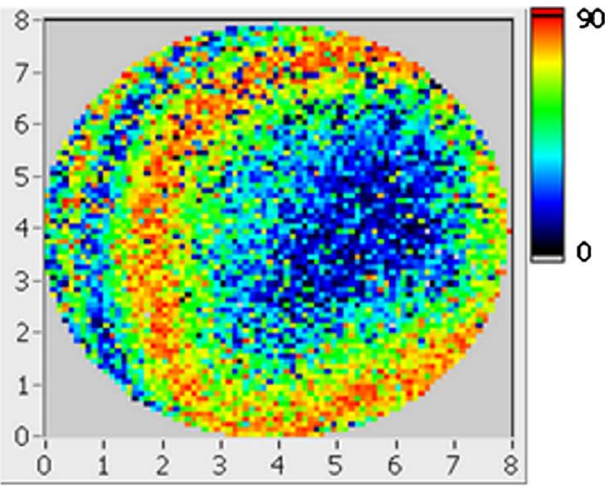

(b)

Fig. 12 (a) Slow-axis orientation and (b) retardation of the in vitro back surface of the tilted (30 deg) cornea measured by PS-OCT; $x$ and $y$ axes in millimeters; color scale in degrees. (Images taken from Ref. 14.) (Color online only.)

measured PS-OCT retardation patterns. The retardation patterns of the PS-OCT images [Figs. 8(b) and 9(b)] show greater values than the simulations [Fig. 5(b)] and also oscillations at the corneal periphery. This effect is due to the algorithm. Values over $90 \mathrm{deg}$ are wrapped, causing ambiguity. In Fig. 5(b), the maximum value is around $45 \mathrm{deg}$. However, the corneal retardation measured by PS-OCT is larger. We increased corneal birefringence to $4 \times 10^{-3}$ for simulations. The resulting corneal retardance is shown in Fig. 10. The pattern is now more circularly symmetric. It also shows the peripheral oscillations due to the wrapping procedure. The biaxial-like behavior is now much more hidden. We rescaled the image as before, and made a zoom of the central area (Fig. 11). The biaxial-like behavior can be appreciated, with two low retardance areas. This is in agreement with the layered structure of uniaxial birefringent crystals at each corneal lamella. The same birefringence increase was applied to the horizontalvertical case, but the retardation showed no difference.

In a previous study ${ }^{14}$ in vitro images of the tilted cornea were also acquired with the time domain PS-OCT system. These results are shown in Fig. 12. The cornea was tilted approximately $30 \mathrm{deg}$ (the apex in the figure is displaced to the left along the $x$ axis). The optic axis orientation [Fig.

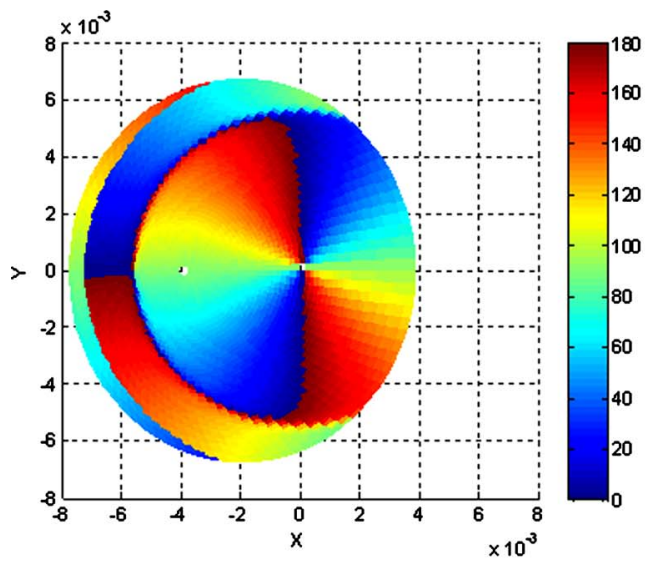

(a) 12(a)] shows an angular rotating pattern centered at the point of the corneal surface that is closest to the instrument. This behavior is similar to that of the nontilted cornea, but the central point of the angular rotation was the apex. Retardation [Fig. 12(b)] shows a low-retardation area around the highest point. Retardation increases forming a circular-similar pattern. There are some oscillations at the left, corresponding to the wrapping of the algorithm. The optic axis orientation [Fig. 12(a) at the left] changes by $90 \mathrm{deg}$ as a consequence. We performed new simulations of the polarization properties of the tilted cornea. The results for the circular-radial case are shown in Fig. 13. The optic axis orientation [Fig. 13(a)] presents an angular distribution centred at the closest to the instrument point. That is the same result of the measurements [Fig. 12(a)]. There is also a 90-deg change at the left of the image due to the wrapping of retardation values over $90 \mathrm{deg}$ [Fig. 13(b)]. Retardation in this figure shows a low-retardation area near the highest point of the cornea. Retardation increases to the left of the image. The resulting pattern is circular with some oscillations due to wrapping. The behavior of the circular-radial case is consequently coincident with the in vitro experimental results for the tilted cornea. The horizontalvertical case (results not shown here) shows a great low-

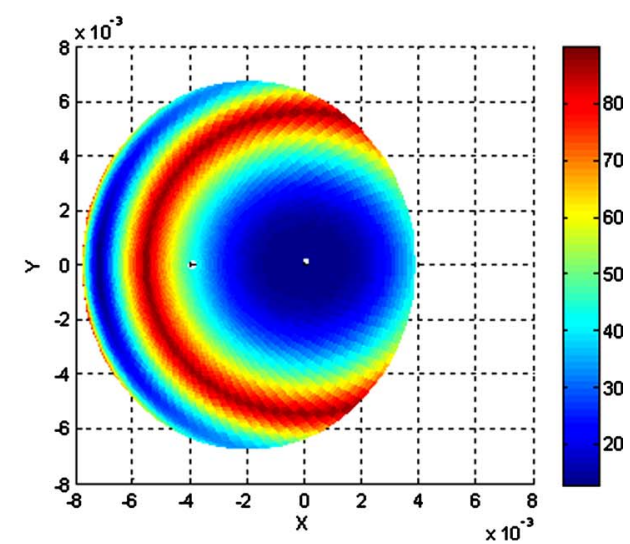

(b)

Fig. 13 Calculated polarization parameters at posterior corneal surface in the tilted case (30 deg). (a) Slow axis orientation and (b) retardation for the circular-radial case. Color scale: degrees. (Color online only.) 
retardation area for the tilted case. The slow-axis orientation shows an irregular pattern in which there is no clear angular rotation, but rather predominant areas of low and high angular values. Other configurations (circular-vertical, cross-arc) were also tested, but the results are not in agreement with the experiments.

The orientation of the retardation pattern at the central cornea is horizontal in the simulations [Figs. 5(b), 6(b), 7, and 11]. This is due to the assumed laboratory reference system, whose $X$ axis is coincident with the horizontal axis of the images. The nasally downward orientation reported in several papers ${ }^{12,13}$ could be related with the orientation of the instrument or that of the measured corneas, or even with different muscles' stresses that alter slightly the fibrils orientation pattern locally. In Ref. 12, two different corneal types are postulated based on this orientation. A third type is proposed in which there is a great low-retardation area, and it is said to be rather unusual. This could be explained in terms of an increasing birefringence, which influences the retardation pattern making it less biaxial-like and more uniaxial-like, with this low-retardation area. In this study, we measured a total of 12 eyes (both eyes in six healthy volunteers). The polarimetric patterns were rather similar to those shown previously. Fibrils orientation was also measured by x-ray diffraction methods, as stated in the introduction. In general, the papers postulate that in the central cornea (excluding limbus) there are preferred fibril orientations in the directions superior-inferior and nasal-temporal. However, they find the existence of an annulus of fibrils in the limbus area, that they describe as a usual annulus of fibrils, ${ }^{33}$ as an interlaced pattern of fibrils of the cornea and the sclera, ${ }^{7}$ or even as four fibril arcs that make a rhomboidal form in the most recent one. ${ }^{34}$ All these proposed patterns are postulated a posteriori from the experimental measurements. No one is strictly the circular-radial pattern postulated here, but there are some similarities with the model of the annulus of fibrils.

\section{Conclusions}

The polarization properties of the cornea present some controversy. Several papers reported corneal birefringence measured by means of different optical techniques, such as scanning laser polarimetry (SLP), PS-OCT, or polarization microscopy. The previous approaches used for the analysis of the results did not take into account nonnormal incidence or arbitrary fibril orientation. In the case of the cornea, the optical configuration employed in PS-OCT requires the consideration of those cases. We presented an approach that takes into account nonnormal incidence and/or non-surface-parallel fibril orientation. This approach is based on the extended Jones matrix method. The results are further decomposed by the Poincare equivalence theorem. The model was applied to corneal structure with alternating circular-radial and horizontal-vertical preferentially oriented fibrils. In vitro and in vivo corneal slow-axis orientation and retardation were measured by PS-OCT. There is a general biaxial-like behavior in the retardation of the simulations in agreement with several previous reports. The circular-radial case seems to better resemble the experimental data. The retardation pattern of the circular-radial case is also compatible with the biaxial-like behavior in the corneal center. The results of this work con- tribute to the interpretation of the PS-OCT corneal measurements and propose a possible corneal fibril arrangement. This information could be useful for corneal diagnosis by polarization sensitive techniques, such as PS-OCT, e.g., to better understand changes associated with keratoconus. ${ }^{35}$ It could also help to adequately compensate corneal polarization when imaging the retina or the eye fundus in general. The presented model tries to contribute to the explanation of corneal polarimetric properties, in this case, measured by PS-OCT. Previous measurements propose different corneal arrangements and polarimetric properties. The controversy about the results of other methods remains in some specific aspects, as stated in the previous paragraph. Further research is in progress.

\section{Acknowledgments}

This work was partially supported by the project TEC200606548/TCM of the Spanish Ministry of Education and Science and by the Austrian Science Fund (FWF Grant No. P19624-B02).

\section{References}

1. M. R. Hee, D. Huang, E. A. Swanson, and J. G. Fujimoto, "Polarization-sensitive low-coherence reflectometer for birefringence characterization and ranging," J. Opt. Soc. Am. B 9, 903-908 (1992).

2. R. W. Knighton, X. R. Huang, and D. S. Greenfield, "Analytical model of scanning laser polarimetry for retinal nerve fiber layer assessment," Invest. Ophthalmol. Visual Sci. 43(2), 383-392 (2002).

3. N. T. Choplin, Q. Zhou, and R. W. Knighton, "Effect of individualized compensation for anterior segment birefringence on retinal nerve fiber layer assessments as determined by scanning laser polarimetry," Ophthalmology 110, 719-725 (2003).

4. M. Pircher, E. Götzinger, B. Baumann, and C. K. Hitzenberger, "Corneal birefringence compensation for polarization sensitive optical coherence tomography of the human retina," J. Biomed. Opt. 12(4), 041210-1, 10 (2007)

5. L. Weiss and R. O. Greep, Histology, McGraw-Hill, New York (1977).

6. Y. Komai and T. Ushiki, "The three-dimensional organization of collagen fibrils in the human cornea and sclera," Invest. Ophthalmol. Visual Sci. 32(8), 2244-2258 (1991).

7. R. H. Newton and K. M. Meek, "The integration of the corneal limbal fibrils in the human eye," Biophys. J. 75, 2508-2512 (1998)

8. A. Daxer and P. Fratzl, "Collagen fibril orientation in the human corneal stroma and its implication in keratoconus," Invest. Ophthalmol. Visual Sci. 38, 121-129 (1997).

9. G. P. Misson, B. H. Timmerman, and P. J. Bryanstron-Cross, "Human corneal stromal lamellar organisation: a polarised light study in pseudophakic eyes," J. Mod. Opt. 55, 625-637 (2008).

10. G. P. Misson, "Circular polarization biomicroscopy: a method for determining human corneal stromal lamellar organization in vivo," Ophthalmic Physiol. Opt. 27, 256-264 (2007).

11. R. A. Bone and G. Draper, "Optical anisotropy of the human cornea determined with a polarizing microscope," Appl. Opt. 46(34), 83518357 (2007).

12. R. W. Knighton, X. Huang, and L. A. Cavuoto, "Corneal birefringence mapped by scanning laser polarimetry," Opt. Express 16(18), 13738-13751 (2008).

13. G. J. Van Blokland and S. C. Verhelst, "Corneal polarization in the living human eye explained with a biaxial model," J. Opt. Soc. Am. A 4(1), 82-90 (1987).

14. E. Götzinger, M. Pircher, M. Sticker, A. F. Fercher, and C. K. Hitzenberger, "Measurement and imaging of birefringent properties of the human cornea with phase-resolved polarization-sensitive optical coherence tomography," J. Biomed. Opt. 9(1), 94-102 (2004).

15. J. M. Bueno and F. Vargas-Martín, "Measurements of the corneal birefringence with a liquid-crystal imaging polariscope," Appl. Opt. 41(1), 116-124 (2002).

16. J. W. Jaronski and H. T. Kasprzak, "Linear birefringence measurements of the in vitro human cornea," Ophthalmic Physiol. Opt. 23, 361-369 (2003). 
17. D. J. Donohue, B. J. Stoyanov, R. L. McCally, and R. A. Farrell, "Numerical modelling of the cornea's lamellar structure and birefringence properties," J. Opt. Soc. Am. A 12(7), 1425-1438 (1995).

18. F. Fanjul-Vélez, M. Pircher, B. Baumann, E. Götzinger, J. L. ArceDiego, and C. K. Hitzenberger, "Modeling human corneal polarization properties and comparison with PS-OCT measurements," in Ophthalmic Technologies XIX, F. Manns, P. G. Söderberg, and A. Ho, Eds., Proc. SPIE 7163, 71630K (2009).

19. F. Fanjul Vélez and J. L. Arce Diego, "Polarimetry of birefringent biological tissues with arbitrary fibril orientation and variable incidence angle," Opt. Lett. 35(8), 1163-1165 (2010).

20. C. K. Hitzenberger, E. Götzinger, M. Sticker, M. Pircher, and A. F. Fercher, "Measurement and imaging of birefringence and optic axis orientation by phase resolved polarization sensitive optical coherence tomography," Opt. Express 9, 780-790 (2001).

21. M. Todorovic, S. Jiao, L. V. Wang, and G. Stoica, "Determination of local polarization properties of biological samples in the presence of diattenuation by use of Mueller optical coherence tomography," Opt. Lett. 29, 2402-2404 (2004).

22. P. Yeh, "Extended Jones matrix method," J. Opt. Soc. Am. 72, 507513 (1982).

23. C. Gu and P. Yeh, "Extended Jones matrix method. II," J. Opt. Soc. Am. A 10, 966-973 (1993).

24. R. A. Farrell, D. Rouseff, and R. L. MacCally, "Propagation of polarized light through two- and three-layer anisotropic stacks," J. Opt. Soc. Am. A 22, 1981-1992 (2005).

25. N. Ugryumova, J. Jacobs, M. Bonesi, and S. J. Matcher, "Novel optical imaging technique to determine the 3-D orientation of collagen fibers in cartilage: variable-incidence angle polarizationsensitive optical coherence tomography," Osteoarthritis Cartilage 17 33-42 (2008).
26. H. Hurwitz and R. C. Jones, "A new calculus for the treatment of optical systems. II. Proof of three general equivalence theorems," $J$. Opt. Soc. Am. 31, 493-499 (1941).

27. E. P. Wigner, Group Theory and its Application to the Quantum Mechanics of Atomic Spectra, Academic Press, New York (1959).

28. J. F. de Boer, T. E. Milner, M. J. C. Van Gemert, and J. S. Nelson, "Two-dimensional birefringence imaging in biological tissue by polarization-sensitive optical coherence tomography," Opt. Lett. 22, 934-936 (1997).

29. E. Götzinger, M. Pircher, and C. K. Hitzenberger, "High speed spectral domain polarization sensitive optical coherence tomography of the human retina," Opt. Express 13, 10217-10229 (2005).

30. B. Baumann, M. Pircher, E. Gotzinger, and C. K. Hitzenberger, "Full range complex spectral domain optical coherence tomography without additional phase shifters," Opt. Express 15, 13375-13387 (2007).

31. C. K. Hitzenberger, A. Baumgartner, W. Drexler, and A. F. Fercher, "Dispersion effects in partial coherence interferometry: implications for intraocular ranging," J. Biomed. Opt. 4, 144-151 (1999).

32. M. Pircher, E. Goetzinger, R. Leitgeb, and C. K. Hitzenberger, "Transversal phase resolved polarization sensitive optical coherence tomography," Phys. Med. Biol. 49, 1257-1263 (2004).

33. R. H. Newton and K. M. Meek, "Circumcorneal annulus of collagen fibrils in the human limbus," Invest. Ophthalmol. Visual Sci. 39, 1125-1134 (1998).

34. H. Aghamohammadzadeh, R. H. Newton, and K. M. Meek, "X-ray scattering used to map the preferred collagen orientation in the human cornea and limbus," Structure 12, 249-256 (2004).

35. E. Götzinger, M. Pircher, I. Dejaco-Ruhswurm, S. Kaminski, C. Skorpik, and C. K. Hitzenberger, "Imaging of birefringent properties of keratoconus corneas by polarization-sensitive optical coherence tomography," Invest. Ophthalmol. Visual Sci. 48, 3551-3558 (2007). 